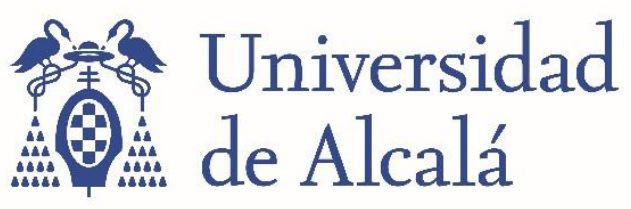

BIBLIOTECA

Document downloaded from the institutional repository of the University of Alcala: http://dspace.uah.es/

This is a postprint version of the following published document:

Filograno, M.L., Corredera, P., González-Herráez, M., RodríguezPlaza, M. and Andrés-Alguacil, A., "Wheel flat detection in high-speed railway systems using fiber Bragg gratings", (May 17, 2011) ; Proc. SPIE 7753, 21st International Conference on Optical Fiber Sensors, 77538D

Available at http://dx.doi.org/10.1117/12.886081

Copyright 2015 Society of Photo Optical Instrumentation Engineers. One print or electronic copy may be made for personal use only. Systematic electronic or print reproduction and distribution, duplication of any material in this paper for a fee or for commercial purposes, or modification of the content of the paper are prohibited.

(Article begins on next page)

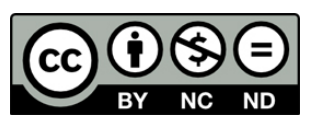

This work is licensed under a

Creative Commons Attribution-NonCommercial-NoDerivatives

4.0 International License. 


\title{
Wheel flat detection in high-speed railway systems using fiber Bragg gratings
}

\author{
M.L. Filograno ${ }^{* a}$, P. Corredera ${ }^{\mathrm{a}}$, M. Gonzalez-Herraez ${ }^{\mathrm{b}}$, \\ M. Rodríguez-Plaza ${ }^{\mathrm{c}}, \mathrm{A}$. Andrés-Alguacil ${ }^{\mathrm{c}}$ \\ anstituto de Óptica, Consejo Superior de Investigaciones Científicas, Madrid, Spain; \\ ${ }^{\mathrm{b}}$ Departamento de Electrónica, Universidad de Alcalá, Alcalá de Henares, Spain; \\ 'Innovation and Technology Direction, Administrador de Infraestructuras Ferroviarias, ADIF, Madrid, Spain.
}

\begin{abstract}
Wheel "flats" are a major source of problems in railway systems since they cause strong wear both in the infrastructure and in the train vehicles. In this work we present field tests concerning the application of Fiber Bragg Grating (FBG) sensors for the detection of out-of-roundness in high-speed train wheels. Wheel flats are shown to cause high-energy impacts on the rails which can be monitored using FBGs located in the rails. The results point out a great potential of this technology for the development of wheel flat detection systems.
\end{abstract}

Keywords: Optical fiber, Bragg gratings, structural monitoring, high speed train, weight in motion, dynamic load, comfort, plane, out-of-roundness, wear, rail.

\section{INTRODUCTION}

Railway traffic is expected to grow very significantly in the next decades. According to recent statistics, the number of passengers will be doubled within 10 years while the volume of goods transported by railway will be tripled. Thus, both axle load and train speed are expected to strongly increase in the next years. In this scenario, wear in the train wheels due to strong braking is also expectable. The most evident consequence of this wear is the appearance of abnormal abrasions in the wheel thread called "flats". Flat spots in the wheel cause strong impacts in the normally smooth power flow from the wheels to the rails. For this reason, wheel flats are a major source of problems in railway systems since they cause strong degradation both in the vehicles and in the infrastructure, they bring noise and discomfort to passengers and they may even cause derailments in extreme cases. There is strong need for inspection techniques to avoid wheel flats, especially in high-speed systems where the tolerances to failure become more stringent ${ }^{1}$. Conventional monitoring systems of wheel flats in railway systems include strain gauge sensors, accelerometers and/or acoustic detection ${ }^{234}$. However, while this sensing technology is well-known and consolidated, it is also expensive, bulky and can be adversely affected by electromagnetic interference. In opposition to these conventional solutions, Fiber Bragg Gratings (FBG) sensors assure immunity to electromagnetic fields (EMI ${ }^{5}$, and can be readily installed at low cost to measure important parameters for the railway engineer in the monitoring of wheel defects and axle load of trains in commercial operation at high speed ${ }^{6}$. This paper reports on field tests about the application of FBG sensing systems to determine the presence of out-of-roundness in train wheels. The field tests are performed on the Spanish high speed line Madrid-Barcelona, with different types of trains. The trains are tested during commercial operation with maximum speeds up to $350 \mathrm{~km} / \mathrm{h}$. The results show the potential of this technology for reliable wheel flat detection using a single FBG installed in the rail.

\section{INSTALLATION OF FBG SENSORS IN THE RAIL}

Wheel flats are known to cause high-energy impacts on the infrastructure. The FBG sensors were therefore installed in the rails. To install the sensors, a straight sector was selected near the Brihuega High Speed maintenance base, around the KP $69+500$ of the Madrid-Barcelona High Speed Line, comprising the transition between an embankment and a trench. The section selected for the tests has a technical building to support the testing campaigns. We selected six different positions of the sensors with respect to the rail. A total number of $20 \mathrm{FBG}$ sensors have been installed, that is 10 per track (see fig. 1 for details of the disposition). Three consecutive spaces between sleepers have been selected. In the

*massimo.filograno@depeca.uah.es; phone +34 91885 6908; mobile +34 693462620 
first space we put four FBG sensors: one in the middle between the two sleepers and adapted to the rail foot - this FBG sensor (S1) is working in pure flexion; a pair of them are adapted to the rail web with an angle with respect to the rail neutral fiber of $45^{\circ}$ - these two FBG sensors (S3 and S4) are working in shear - and the last one (S2) is placed just on the rail neutral fiber in order to check temperature changes in the rail. In the second section between sleepers we use the same sensor structure without the temperature FBG and the third section has a vertical sensor just in the centre of the sleeper (S6) and three flexion sensors placed at different distances between the sleepers (among them, S5). The FBG sensors are directly pasted with an epoxy resin on the rail tracks and are connected by optical cables to an optical fiber

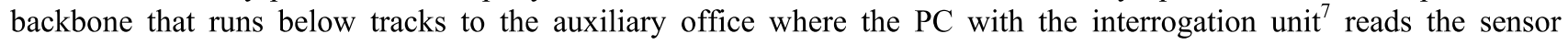
deformation, stores, analyzes the data and displays the train footprints. The installed FBG sensors have been running one year without faults even after snows and extremely hot summer days.

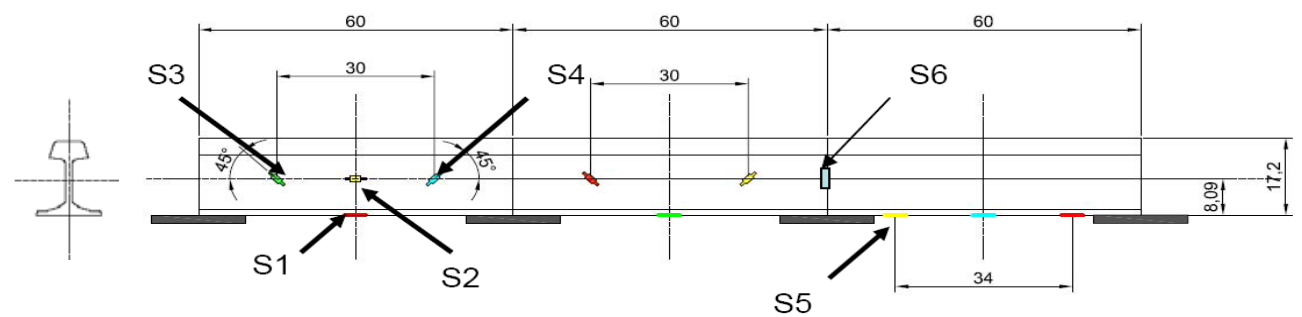

Figure 1: Disposition of 10 FBG sensors along one of the rails.

\section{SHAPE AND SPECTRAL ANALYSIS OF THE SIGNALS}

We have basically six types of dispositions for the FBG strain sensors and every disposition has a different mechanical purpose. For instance, S3 and S4 can determine dynamic load ${ }^{8}$. S6 suffers the maximum compression of all the sensors tested. Even if the following analysis can be obtained similarly with other dispositions, in this paper we focus only on data obtained with flex disposition (more specifically, with sensor S5). Of all the configurations tested we have verified that this is the best option for wheel flat detection with a single sensor since the deformation is sufficiently large and the wheel flats are well visible. Comparative spectral analysis provides us with a measure of the signal bandwidth caused by the deformation of a healthy wheel as a function of the train speed (deformation is faster for faster trains). We found a conservative value of .9 multiplied for the velocity expressed in $\mathrm{km} / \mathrm{h}$, so:

$$
B_{\text {signal }}[\mathrm{Hz}]=.9\left[\frac{\mathrm{h}}{\mathrm{km} \times \mathrm{s}}\right] * V_{\text {train }}\left[\frac{\mathrm{km}}{\mathrm{h}}\right]
$$

In fig. 2-right we show a trace obtained for sensor S5 with the passage of a S-103 SIEMENS-VELARO train (32 axes). A time-frequency analysis (spectrogram) of this signal is shown in fig. 2-left. In the bottom of the spectrogram we can notice the spectral content of the signal and its bandwidth.
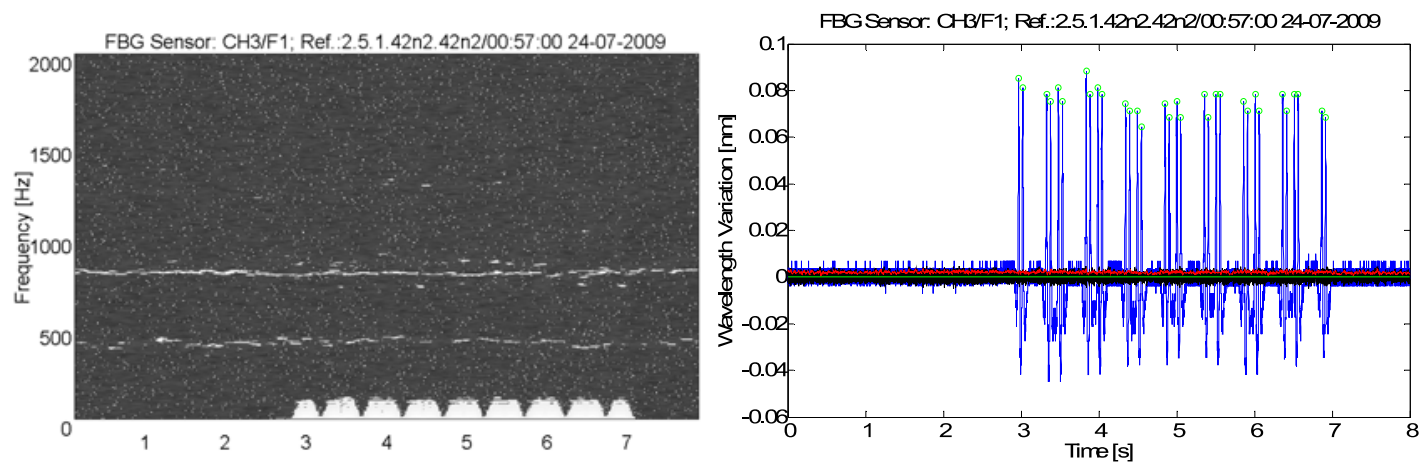

Figure 2: Spectrogram (left) and the trace for a flex-position sensor (right) of an S-103 SIEMENS-VELARO at $49 \mathrm{~m} / \mathrm{s}$.

The two horizontal lines (near $400 \mathrm{~Hz}$ and $800 \mathrm{~Hz}$ ) are two pseudo-harmonics generated by the high gain of the system. This pseudo-harmonics vary from trace to trace but can be easily eliminated since their frequencies change slowly and 
continuously with time and they are visible even when there is no train passing. In this train, all the wheels are healthy and in good state. The fig. 2-right shows in blue the signal obtained by the FBG, in black the high-pass filtered signal $\left(f_{c}=B_{\text {signal }}\right)$, in red the envelope of the filtered signal and finally the green circles mark the time when the wheels cross over the sensor. This is produced by the automatic signal processing done in the PC unit. All these elements are now generated by program in post-processing mode, but can be obtained in real-time-processing mode.
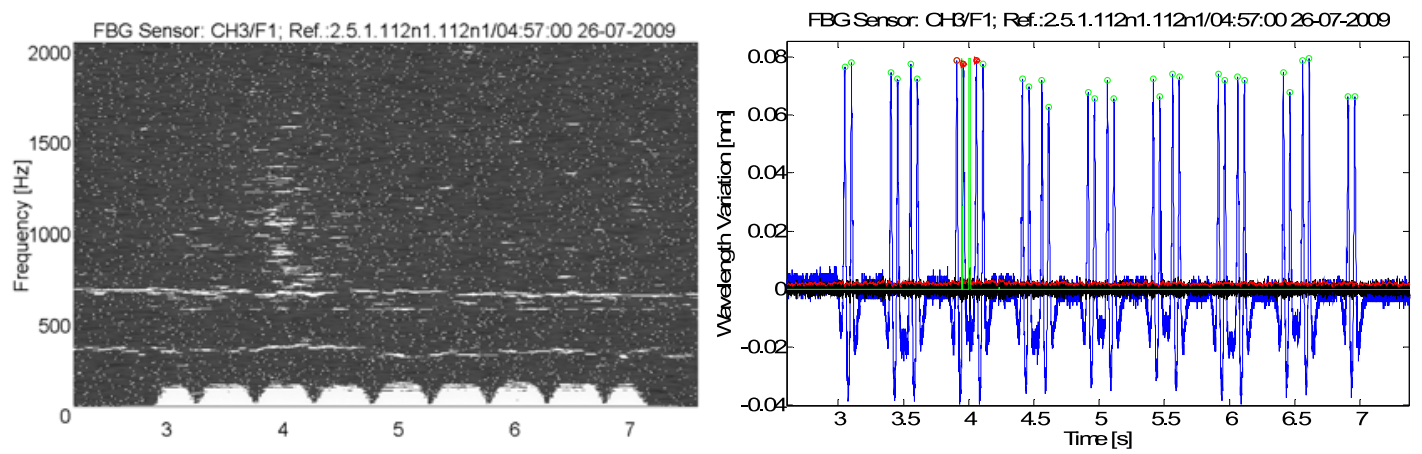

Figure 3: Spectrogram (left) and the trace (right) for an S-103 at $49.5 \mathrm{~m} / \mathrm{s}$ with a single impact generated by a defect in a wheel.

Fig. 3 represents the spectrogram and the trace for the same type of train but with a defect (probably in wheel $8^{\text {th }}$, counting the wheels from the first wagon that is in the left side of the graph).
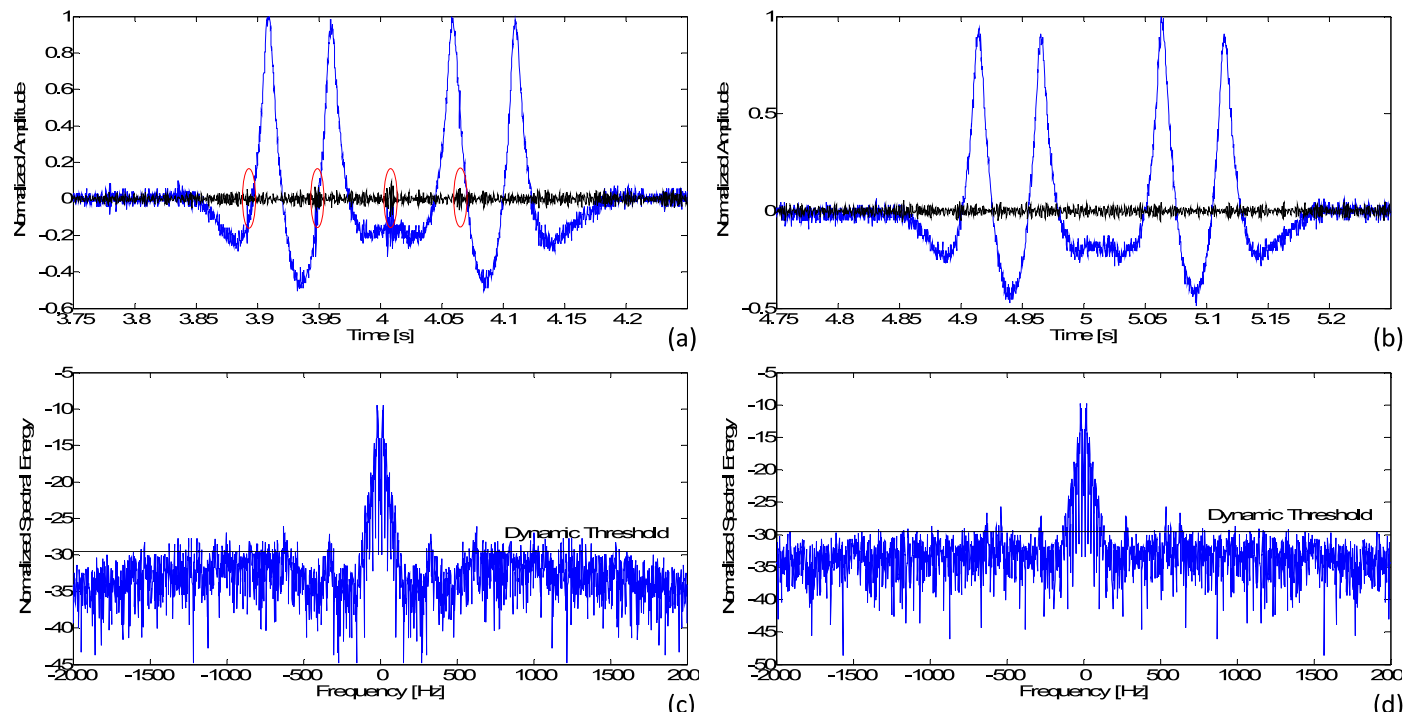

Figure 4 - Time domain and spectral analysis details of fig. 3: a) deformation (blue) and high-pass filtered signal (black) while flatted-wheel pass ; b) deformation (blue) and high-pass filtered signal (black) while good-health-state wheel pass; c) FFT of the deformation while flatted-wheel pass; d) FFT of the deformation while good-health wheel pass.

We can see significant high-frequency associated to the passage of this wheel. In the trace, the program displays this defect is with red circles in the wheels (instead of green circles). Out-of-roundness in the wheel generates relevant highfrequency spectral components out of the signal bandwidth previously obtained (see fig. 3-left and fig. 4c, d). For this reason the impact detection can be simply obtained checking the level of energy in the high pass filtered signal (red marker in fig. 4a). In our case we use the envelope of the high-pass filtered signal to obtain an estimation of this energy. To do a correct detection we need to calculate a threshold level of energy (including noise) for the high-pass signal. This threshold value is obtained from the envelope of the high-pass signal while there is no train (baseline noise). For this detection procedure it is important to acquire the signals with a sampling frequency much larger than $B_{\text {signal }}$. For the train speed in this sector, a sampling frequency of at least $4 \mathrm{kHz}$ is necessary. When the energy in the high-pass filtered signal 
exceeds a threshold we consider to have a significant out-or roundness in the wheel. Our preliminary observations indicate that with this system, a 70\% increase in the energy of the high-pass signal (with respect to the baseline noise) gives a good estimation of significant wheel defects. Focusing in the time position of the red markers of fig. 4a we can observe that a perfect plane in a wheel generates a periodical perturbation with a frequency of repetition related to the velocity of the train and the diameter of the wheel:

$$
f_{p}[H z]=\frac{V_{\text {train }}\left[\frac{m}{s}\right]}{\pi D[m]}
$$

The condition of a single and clear plane perfectly visible in a trace is a relatively rare hypothesis; the common case is to have one or more generally defective wheels. Energy created by the vibrations of a flat wheel normally flow to the near wheels through the shock absorbers and the vibrations of the wagon (that reduce the comfort of the passengers). These vibrations pass also through the rail. This causes the detection of imperfections not only in the affected wheel but also in positions close to the really defective wheel. In terms of repetitiveness, during our campaign of tests we found that the detection of the defective wheels was repetitive for the same train in different days. This can be considered as a confirmation of the repetitiveness of the measurement system and the signal processing algorithm.

\section{CONCLUSIONS}

In conclusion, we have shown that fiber optic sensing technology can be adequately used for robust detection of wheel defects in high-speed railway systems. Wheels with defects can wear the rail, reduce the comfort of the passengers and in some extreme cases cause train derailment. Undoubtedly a fiber-optic solution to this problem is close to ideal, since noise problems are eliminated and the identification of the defective wheels is significantly more reliable than using conventional technology, strongly affected by electromagnetic noise. We have shown here that wheel flats can be determined with a single FBG sensor.

\section{REFERENCES}

[1] Ho, S.L. Lee, K.K. Lee, K.Y. Tam, H.Y. Chung, W.H. Liu, S.Y. Yip, C.M. Ho, T.K. 'A Comprehensive Condition Monitoring of Modern Railway'; Railway Condition Monitoring, 2006. The Institution of Engineering and Technology International Conference - Birmingham ISBN: 086341-732-9.

[2] Brant Stratman, Yongming Liu, Sankaran Mahadevan 'Structural Health Monitoring of Railroad Wheels Using Wheel Impact Load Detectors'; Journal of Failure Analysis and Prevention Volume 7, Number 3, 218-225, DOI: 10.1007/s1 1668-007-9043-3.

[3] Kyowa Flat Wheel Detection System www.kyowa-ei.co.jp

[4] www.gotchamonitoringsystem.com

[5] Hwa-yaw Tam, Applications of fibre Bragg grating sensors in railways; Opto-Electronics and Communications Conference, 2008 and the 2008 Australian Conference on Optical Fibre Technology. OECC/ACOFT 2008. ISBN: 978-0-85825-807-5.

[6] S.L. Ho, K.Y. Lee, K.K. Lee, H.Y. Tam, W.H. Chung, S.Y. Liu, C.M. Yip and T.K. Ho, 'A comprehensive condition monitoring of modern railway', IET Intl. Conf. on Railway Condition Monitoring, pp.125-129, (2006).

[7] www.fibersensing.com

[8] M. L. Filograno, A. Rodríguez-Barrios, M. González-Herraez, P. Corredera, S. Martín-López, M. Rodríguez-Plaza, A. Andrés-Alguacil, 'Real time monitoring of railway traffic using Fiber Bragg Grating sensors' - Proceedings of the 2010 Joint Rail Conference JRC2010. 